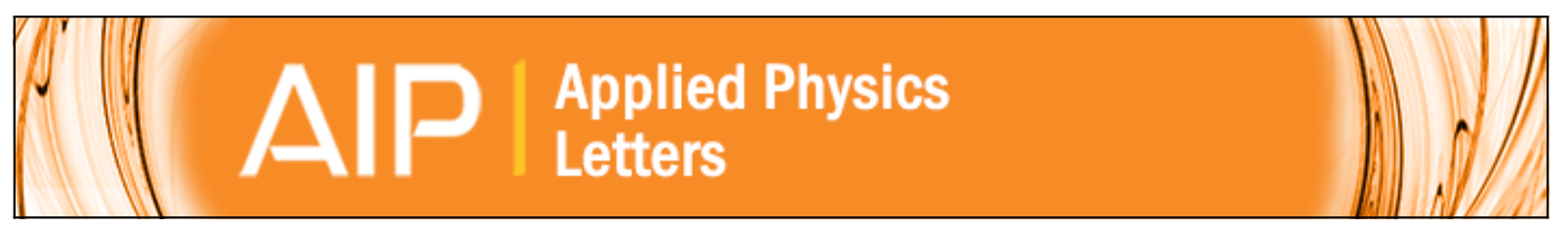

Quantum capacitance in topological insulators under strain in a tilted magnetic field M. Tahir and U. Schwingenschlögl

Citation: Applied Physics Letters 101, 231609 (2012); doi: 10.1063/1.4770000

View online: http://dx.doi.org/10.1063/1.4770000

View Table of Contents: http://scitation.aip.org/content/aip/journal/apl/101/23?ver=pdfcov

Published by the AIP Publishing 


\title{
Quantum capacitance in topological insulators under strain in a tilted magnetic field
}

\author{
M. Tahir and U. Schwingenschlögl ${ }^{\text {a) }}$ \\ PSE Division, KAUST, Thuwal 23955-6900, Kingdom of Saudi Arabia
}

(Received 16 October 2012; accepted 20 November 2012; published online 6 December 2012)

\begin{abstract}
Topological insulators exhibit unique properties due to surface states of massless Dirac fermions with conserved time reversal symmetry. We consider the quantum capacitance under strain in an external tilted magnetic field and demonstrate a minimum at the charge neutrality point due to splitting of the zeroth Landau level. We also find beating in the Shubnikov de Haas oscillations due to strain, which originate from the topological helical states. Varying the tilting angle from perpendicular to parallel washes out these oscillations with a strain induced gap at the charge neutrality point. Our results explain recent quantum capacitance and transport experiments. (C) 2012 American Institute of Physics. [http://dx.doi.org/10.1063/1.4770000]
\end{abstract}

In recent years, progress in the realization of topological insulators (TIs) has attracted much interest in condensed matter physics. ${ }^{1-12}$ In general, due to strong spin-orbit interaction, TIs have an energy gap in the bulk and gapless conducting surface states. By the conserved time reversal symmetry these surface states are immune to defects, nonmagnetic impurities, and other environmental perturbations, paving the way to non-dissipative applications. The dispersion is characterized by conical branches touching at a high symmetry point of the first Brillouin zone. The surface states of the compounds $\mathrm{Bi}_{2} \mathrm{Se}_{3}, \mathrm{Sb}_{2} \mathrm{Te}_{3}$, and $\mathrm{Bi}_{2} \mathrm{Te}_{3}$ are chiral two-dimensional non-ideal Dirac fermions. ${ }^{8}$ Dirac fermions can be seen in angle resolved photoemission experiments ${ }^{9,10,13-16}$ and energy quantization of the surface states has been confirmed by scanning tunneling spectroscopy. ${ }^{17,18}$ Along the same line, a two dimensional TI state ${ }^{19,20}$ was first predicted and observed in HgTe quantum wells. ${ }^{2,3}$ Transport measurements have demonstrated a vanishing bulk contribution to the electronic states in two dimensional HgTe. ${ }^{21}$ More recently, transport measurements of a $70 \mathrm{~nm}$ thick $\mathrm{HgTe}$ film in a perpendicular magnetic field ${ }^{22}$ have explained the Rashba-like splitting of the electronic bands. ${ }^{10}$ This splitting is due to the strain induced by the substrate, which breaks the inversion symmetry and results in an energy gap at the TI surface. ${ }^{4,23}$

Our study is motivated by recent experiments on the $\mathrm{Bi}_{2} \mathrm{Se}_{3}$ quantum capacitance ${ }^{12}$ and $\mathrm{HgTe}$ magnetotransport. ${ }^{22}$ The quantum capacitance is an important tool for accessing the electronic properties of solid state systems. Capacitance measurements have advantages over traditional transport measurements in probing the thermodynamic density of states of an electron system, ${ }^{24}$ since they are less complicated and sensitive to scattering details. Though TI research has focused on transport properties, gaining insight into the fundamental electronic properties and device physics calls for a study of the capacitance-voltage characteristics. In view of this, more attention is now being paid to electrostatic properties such as the quantum capacitance. The present

a)udo.schwingenschlogl@kaust.edu.sa. Tel.: +966(0)544700080. work therefore explains experimental results ${ }^{12}$ and predicts the effects of strain on the quantum capacitance of TIs.

An effective model to describe Dirac fermions on the surface of a three dimensional TI is considered. The two dimensional Dirac-like Hamiltonian incorporating strain effects in a tilted magnetic field is ${ }^{10,22,25}$

$$
H=\tau_{z} v\left(\sigma_{x} \boldsymbol{\Pi}_{y}-\sigma_{y} \boldsymbol{\Pi}_{x}\right)+\tau_{z} \Delta \mathbf{I} .
$$

Here, $\mathbf{I}$ is the identity matrix, $\sigma_{x}$ and $\sigma_{y}$ are Pauli matrices that operate on the real spin space, $\tau_{z}=+/-$ defines the upper and lower surfaces of the TI, $v$ denotes the Fermi velocity of the Dirac fermions, $\boldsymbol{\Pi}=\mathbf{p}+e \mathbf{A} / c$ is the two-dimensional canonical momentum with vector potential $\mathbf{A}$, and $c$ is the speed of light. In addition, the strain energy $\Delta$ represents the inversion symmetry breaking. We employ the Landau gauge and express the vector potential as $\mathbf{A}=(0, x B \cos \theta-z B \sin \theta, 0)$, where $z$ is the TI film thickness. The energy of the Landau level $n$ is obtained as

$$
\begin{aligned}
& E_{0}^{\tau_{z}}=\tau_{z} \Delta, \quad n=0 \\
& E_{n, \lambda}^{\tau_{z_{z}}}=\lambda \hbar \omega \sqrt{n}+\tau_{z} \Delta, \quad n \neq 0
\end{aligned}
$$

with $\lambda=+/-$ for the electron and hole bands. Moreover, $\tau_{z} \Delta$ is the splitting of the levels due to the strain and $\omega=v \sqrt{2 e B \cos \theta / \hbar}$ is the cyclotron frequency of the Dirac fermions, which depends on the tilting angle.

We consider a gated TI device where the capacitance is formed between the gate and a thin TI. The quantum capacitance $C_{Q}$, i.e., the charge response in the channel as the channel potential is varied, is large in TIs, in fact the dominant capacitive contribution, and thus is an important quantity for device design. It can be written as $C_{Q}=e^{2} D_{T}(B),{ }^{24,26}$ where $D_{T}(B)$ is the temperature dependent density of states at finite magnetic field and can be determined from the relation,

$$
D_{T}(B)=\frac{\partial n_{c}}{\partial E_{F}}=\int_{0}^{\infty} d \varepsilon \frac{\partial f\left(E-E_{F}\right)}{\partial E_{F}} D(E) .
$$

Here, $n_{c}$ is the carrier concentration, $E_{F}$ the Fermi energy, and $f(x)$ the Fermi Dirac distribution function. In the limit of 
zero temperature, we have $D_{T}(B)=D\left(E_{F}\right)$, where the DOS is defined as $D(E)=\frac{1}{2 \pi l^{2}} \sum_{n, \tau_{z}, \lambda} \delta\left(E-E_{n, \lambda}^{\tau_{z}}\right)$ and $l$ $=\sqrt{\hbar / e B \cos \theta}$ is the magnetic length. Assuming a Gaussian broadening (width $\Gamma$, zero shift) of the Landau levels, the density of states per unit area is

$$
\begin{aligned}
D\left(E_{F}\right)= & \frac{1}{\pi l^{2}}\left\{\sum_{\tau_{z}} \frac{1}{\Gamma \sqrt{2 \pi}} \exp \left[-\frac{\left(E_{F}-E_{0}^{\tau_{z}}\right)^{2}}{2 \Gamma^{2}}\right]\right. \\
& \left.+\sum_{\lambda, \tau_{z}} \sum_{n=1}^{\infty} \frac{1}{\Gamma \sqrt{2 \pi}} \exp \left[-\frac{\left(E_{F}-E_{n, \lambda}^{\tau_{z}}\right)^{2}}{2 \Gamma^{2}}\right]\right\} .
\end{aligned}
$$

At the charge neutrality point $\left(E_{F}=0\right)$, we obtain

$$
\begin{aligned}
D\left(E_{F}=0\right)= & \frac{1}{\pi l^{2}} \sum_{\tau_{z}} \frac{1}{\Gamma \sqrt{2 \pi}} \exp \left[-\frac{\left(\tau_{z} \Delta\right)^{2}}{2 \Gamma^{2}}\right] \\
& \times\left\{1+2 \sum_{n=1}^{\infty} \exp \left[-\frac{(\hbar \omega \sqrt{n})^{2}}{2 \Gamma^{2}}\right]\right\},
\end{aligned}
$$

which can be written as

$$
D\left(E_{F}=0\right)=\frac{4 \Gamma}{v^{2} \hbar^{2} \pi \sqrt{2 \pi}} \exp \left[-\frac{\Delta^{2}}{2 \Gamma^{2}}\right]\left\{\frac{\chi}{\tanh \chi}\right\}
$$

with $\chi=\left(\frac{\hbar \omega}{2 \Gamma}\right)^{2}$. Equation (6) applies to any magnetic field, also to zero field for which we have $\frac{\chi}{\tanh \gamma}=1$. Clearly, there is an energy gap at the charge neutrality point. We note that $C_{Q}$ at the charge neutrality point reduces to the result obtained in the absence of strain and tilting. ${ }^{24}$

We next show $C_{Q}$ in Fig. 1 as a function of the Fermi energy (i.e., of the gate voltage) to address the splitting (at the charge neutrality point) and beating (for higher Landau levels), which are caused by the strain induced contribution to the energy, see Eq. (2). The following parameters are employed: ${ }^{10,12,22} \Delta=4.2 \mathrm{meV}, \quad B=3 \mathrm{~T}, \quad \theta=0^{\circ}, n_{c}=5$ $\times 10^{15} \mathrm{~m}^{-2}$, and $v=3 \times 10^{5} \mathrm{~ms}^{-1}$. In order to make the analytical calculation tractable, we chose a constant level width of $\Gamma=0.7 \mathrm{meV}$. We see in Fig. 1 that there is a minimum in $C_{Q}$ at $E_{F}=0$ and that higher Landau levels show a beating pattern. The latter traces back to mixing of neighboring

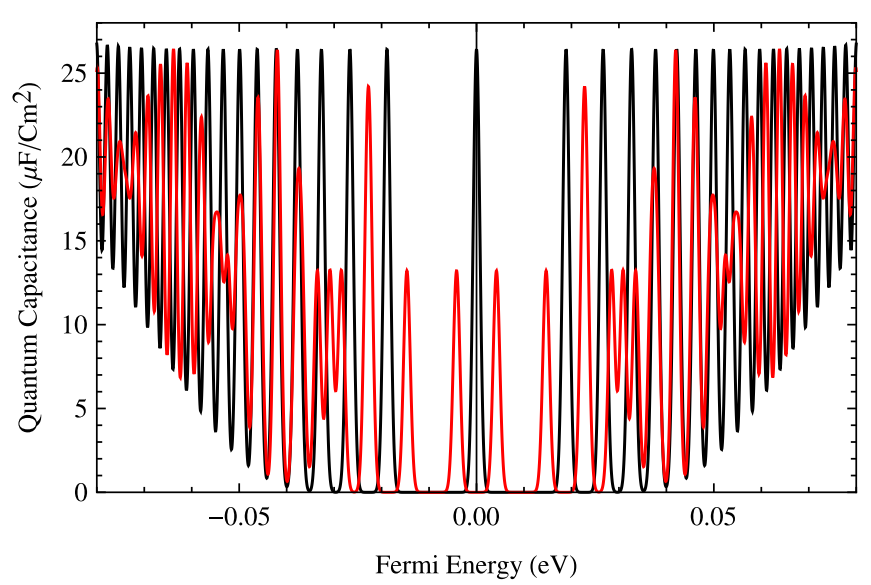

FIG. 1. Quantum capacitance as a function of the Fermi energy at $B=3 \mathrm{~T}$, $\Delta=4.2 \mathrm{meV}, \theta=0^{\circ}$, and $\Gamma=0.7 \mathrm{meV}$. Black and red lines correspond to zero and finite strain energy, respectively.
Landau levels, leading to two unequally spaced energy branches in accordance with Eq. (2). The zeroth Landau level splits into two levels with equal weights for the electron and hole contributions, while all other Landau levels show a well resolved beating pattern. In order to observe this effect, the broadening of the Landau levels must be less than the strain energy. To better understand the splitting and beating pattern, the frequency of the magnetic oscillations can be explored using the Poisson summation formula. We obtain for Gaussian broadening of the DOS for the $n \geq 1$ Landau levels,

$$
\begin{aligned}
D\left(E_{F}\right)= & \frac{\left|E_{F}\right|}{\pi \hbar^{2} v^{2}}\left\{1+2 \sum_{\tau_{z}} \sum_{k=1}^{\infty}(-1)^{k} \exp \left[-\left(\frac{2 \pi k E_{F} \sqrt{2} \Gamma}{\hbar^{2} \omega^{2}}\right)^{2}\right]\right. \\
& \left.\left.\times \cos \left[\frac{2 \pi k}{\hbar^{2} \omega^{2}}\left(E_{F}-\tau_{z} \Delta\right)^{2}\right)\right]\right\} .
\end{aligned}
$$

For $\Gamma \gg \hbar \omega$, it is sufficient to retain only the first order term $(k=1)$, since contributions of the higher order terms are highly damped. Equation (7) gives us the frequency of the beating of the $\mathrm{SdH}$ oscillations for different tilting (as the cyclotron frequency $\omega$ depends on the field). Nodes occur when the oscillatory part of Eq. (7) equals zero,

$$
\sum_{\tau_{z}} \cos \left[\frac{2 \pi}{\hbar^{2} \omega^{2}}\left(E_{F}-\tau_{z} \Delta\right)^{2}\right]=0
$$

This can be simplified to

$$
2 \cos \left[\frac{2 \pi E_{F}^{2}}{\hbar^{2} \omega^{2}}\right] \cos \left[\frac{4 \pi E_{F}}{\hbar^{2} \omega^{2}} \Delta\right]=0
$$

representing a wave with $\mathrm{SdH}$ frequency whose amplitude oscillates at a lower frequency. This results in a beating pattern when $\Delta \ll E_{F}$. The $\mathrm{SdH}$ frequency given by the first cosine term in Eq. (9) depends on the tilting angle as $\hbar^{2} \omega^{2}=v^{2} \hbar(2 e B \cos \theta)$. The amplitude of the $\mathrm{SdH}$ oscillations is modulated by $\cos \left[\frac{4 \pi E_{F}}{\hbar^{2} \omega^{2}} \Delta\right]$ such that nodes occur at $\frac{4 E_{F} \Delta}{\hbar^{2} \omega^{2}}= \pm 0.5, \pm 1.5, \ldots$. We note that the amplitude modulation occurs when strain is present in the system. Moreover, the threshold magnetic field where beating is seen depends on the strain energy.

We can conclude that strain plays the main role in the splitting of the $\mathrm{SdH}$ oscillations and formation of beating patterns in quantum capacitance measurements. The former appear in the high and the latter in the low magnetic field regime, as shown in Fig. 2. Of course, splitting of the Landau levels is also important for many other physical quantities besides the quantum capacitance. Our results for $\theta=0$ are in excellent agreement with recent $\mathrm{HgTe}$ experiments under strain for low to high magnetic fields. ${ }^{22}$ The Boltzmann transport equation for short range scattering can be obtained from the Einstein relation in terms of the previously discussed density of states as $\sigma_{x x}=e^{2} v_{F}^{2} \tau D_{T}(B)$, where $\tau$ is the relaxation time. Both phenomena described in Ref. 22, splitting and beating, thus are explained by our theory.

Finally, we compare our results to the experimental observations for the quantum capacitance in Ref. 12 for 


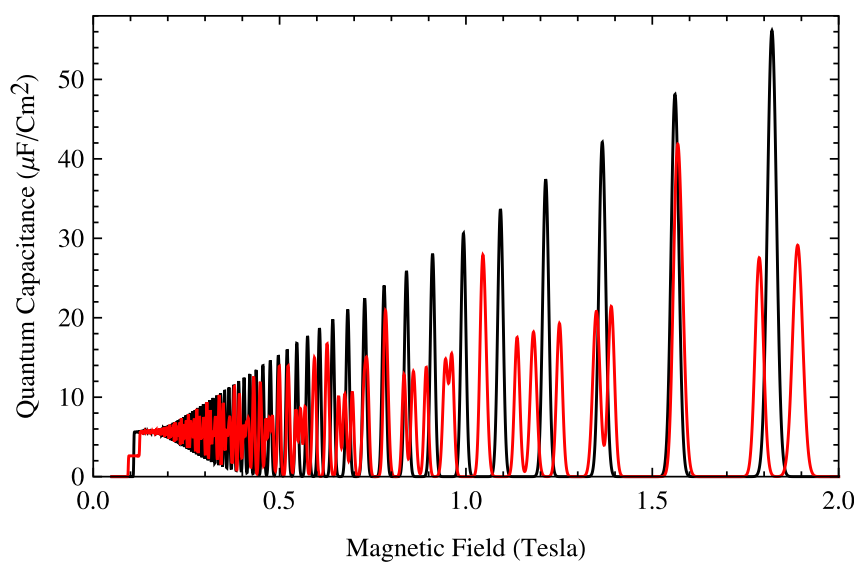

FIG. 2. Quantum capacitance as a function of the magnetic field. Black and red lines correspond to zero and finite strain energy, respectively. We use $\Delta=4.2 \mathrm{meV}, \theta=0^{\circ}$, and $\Gamma=0.2 \mathrm{meV}$.

$\Delta=0$. We show $C_{Q}$ in Fig. 3 as a function of $E_{F}$ (i.e., the gate voltage) for different tilting angles. The $\mathrm{SdH}$ oscillations are suppressed when the angle grows, since we are decreasing the perpendicular component of the magnetic field. When the magnetic field is almost completely aligned with the surface, $\theta \gtrsim 80^{\circ}$, the $\mathrm{SdH}$ oscillations are washed out. To explain this behavior, we study the period of the $\mathrm{SdH}$ oscillations as a function of the tilt angle. Neglecting strain effects, the tilt angle dependence results from the cosine term in Eq. (7) as

$$
\Delta\left(B^{-1}\right)=\frac{e \cos \theta}{h n_{c}} .
$$

When the magnetic field is fixed and the sample is rotated as in the experiment in Ref. 12 then the SdH maxima and minima change, which is shown in Fig. 3. According to Eq. (10), the oscillations are washed out completely for $\theta=90^{\circ}$. We find that the results in Fig. 3 are consistent with the experimental Fig. 4 in Ref. 12. The fact that the strain induced splitting and beating depends on the effective magnetic field, i.e., the tilt angle, causes a complex pattern of $\mathrm{SdH}$ oscillations, which we discuss next.

For finite strain, we show $C_{Q}$ as a function of $E_{F}$ for different tilting angles in Fig. 4. As the tilting angle increases,

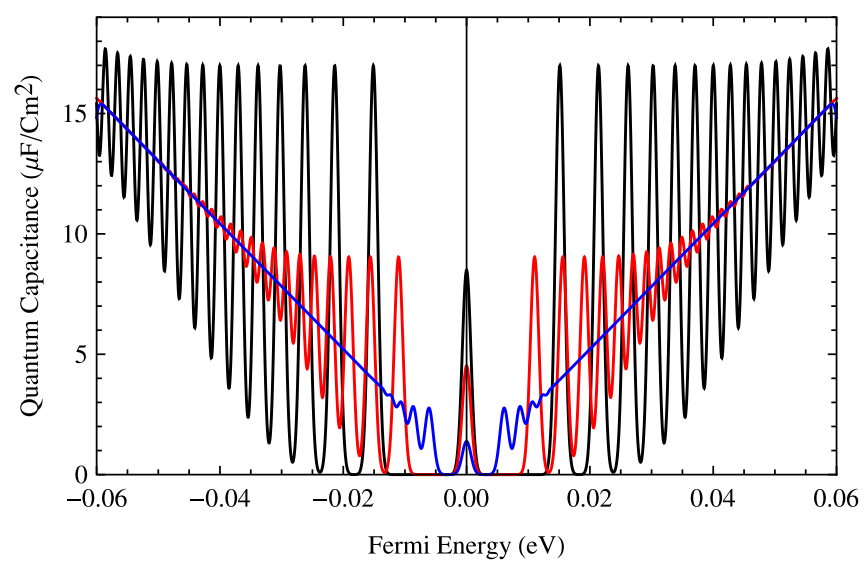

FIG. 3. Quantum capacitance as a function of the Fermi energy. Black, red, and blue lines correspond to tilting angles of $50^{\circ}, 70^{\circ}$, and $85^{\circ}$, respectively. All other parameters are same as in Fig. 1.

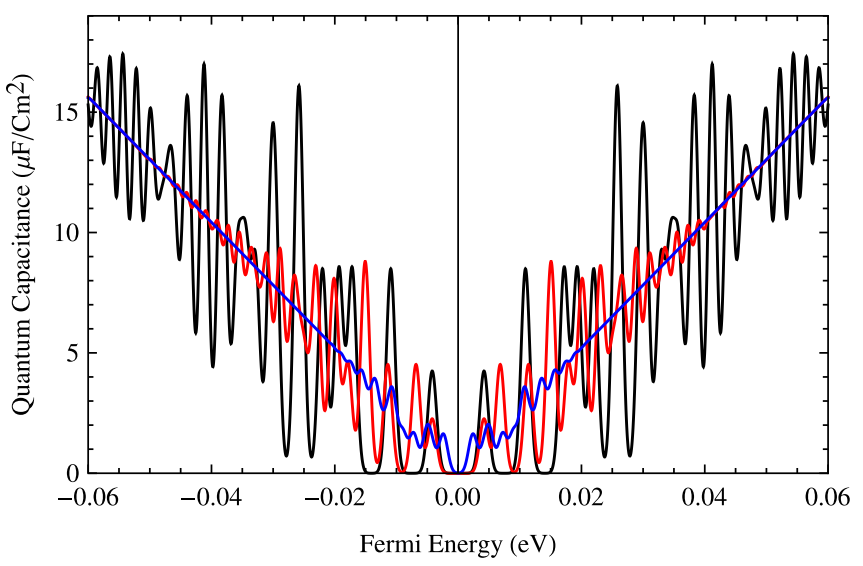

FIG. 4. Quantum capacitance as a function of the Fermi energy. Black, red, and blue lines correspond to tilting angles of $50^{\circ}, 70^{\circ}$, and $85^{\circ}$, respectively. All other parameters are same as in Fig. 1.

the beating pattern of the $\mathrm{SdH}$ oscillations shifts from high to low $E_{F}$ and vanishes when the magnetic field is fully aligned with the surface. The strain induced energy gap at the charge neutrality point is not affected by the direction of the magnetic field. Moreover, the tilted magnetic field mixes the upper and lower surface states, whereas the strain mixes neighbouring Landau levels. The described effects may be confirmed by experiments similar to those in Ref. 12 when strain is incorporated.

In conclusion, we have investigated the effects of strain induced symmetry breaking on the quantum capacitance of TIs in a tilted magnetic field. It turns out that splitting of the Landau levels under strain leads to a minimum in the quantum capacitance at the charge neutrality point. Evidence for metal to insulator transitions and beating of the $\mathrm{SdH}$ oscillations is found. A splitting occurs when the subband broadening is much smaller than the Landau level separation (high magnetic field regime), whereas we obtain a beating pattern when the subband broadening is of comparable size (weak magnetic field regime). Furthermore, when the magnetic field changes from perpendicular to parallel the $\mathrm{SdH}$ oscillations are washed out. However, the strain induced gap at the charge neutrality point remains unaffected.

${ }^{1}$ C. L. Kane and E. J. Mele, Phys. Rev. Lett. 95, 226801 (2005).

${ }^{2}$ B. A. Bernevig, T. L. Hughes, and S. C. Zhang, Science 314, 1757 (2006).

${ }^{3}$ M. König, S. Wiedmann, C. Brüne, A. Roth, H. Buhmann, L. Molenkamp, X.-L. Qi, and S.-C. Zhang, Science 318, 766 (2007).

${ }^{4}$ L. Fu and C. L. Kane, Phys. Rev. B 76, 045302 (2007).

${ }^{5}$ L. Fu, C. L. Kane, and E. J. Mele, Phys. Rev. Lett. 98, 106803 (2007).

${ }^{6}$ J. E. Moore and L. Balents, Phys. Rev. B 75, 121306 (2007).

${ }^{7}$ D. Hsieh, D. Qian, L. Wray, Y. Xia, Y. S. Hor, R. J. Cava, and M. Z. Hasan, Nature (London) 452, 970 (2008).

${ }^{8}$ H. Zhang, C.-X. Liu, X.-L. Qi, X. Dai, Z. Fang, and S.-C. Zhang, Nature Phys. 5, 438 (2009).

${ }^{9}$ Y. Xia, D. Qian, D. Hsieh, L. Wray, A. Pal, H. Lin, A. Bansil, D. Grauer, Y. Hor, R. Cava, and M. Z. Hasan, Nat. Phys. 5, 398 (2009).

${ }^{10}$ Y. Zhang, K. He, C. Z. Chang, C. L. Song, L. L. Wang, X. Chen, J. F. Jia, Z. Fang, X. Dai, W. Y. Shan, S. Q. Shen, Q. Niu, X. L. Qi, S. C. Zhang, X. C. Ma, and Q. K. Xue, Nat. Phys. 6, 584 (2010).

${ }^{11}$ M. Z. Hasan and C. L. Kane, Rev. Mod. Phys. 82, 3045 (2010).

${ }^{12}$ F. Xiu, N. Meyer, X. Kou, L. He, M. Lang, Y. Wang, X. Yu, A. V. Fedorov, J. Zou, and K. L. Wang, Sci. Rep. 2, 669 (2012).

${ }^{13}$ Y. L. Chen, J.-H. Chu, J. G. Analytis, Z. K. Liu, K. Igarashi, H.-H. Kuo, X. L. Qi, S. K. Mo, R. G. Moore, D. H. Lu, M. Hashimoto, T. Sasagawa, S. C. Zhang, I. R. Fisher, Z. Hussain, and Z. X. Shen, Science 329, 659 (2010). 
${ }^{14}$ M. Bianchi, D. Guan, S. Bao, J. Mi, B. B. Iversen, P. D. C. King, and P. Hofmann, Nat. Commun. 1, 128 (2010).

${ }^{15}$ J. Zhang, C. Z. Chang, Z. Zhang, J. Wen, X. Feng, K. Li, M. Liu, K. He, L. Wang, X. Chen, Q. K. Xue, X. Ma, and Y. Wang, Nat. Commun. 2, 574 (2011).

${ }^{16}$ T. Arakane, T. Sato, S. Souma, K. Kosaka, K. Nakayama, M. Komatsu, T. Takahashi, Z. Ren, K. Segawa, and Y. Ando, Nat. Commun. 3, 636 (2012).

${ }^{17}$ Z. Alpichshev, J. G. Analytis, J.-H. Chu, I. R. Fisher, Y. L. Chen, Z. X. Shen, A. Fang, and A. Kapitulnik, Phys. Rev. Lett. 104, 016401 (2010).

${ }^{18}$ P. Cheng, C. Song, T. Zhang, Y. Zhang, Y. Wang, J. F. Jia, J. Wang, Y. Wang, B. F. Zhu, X. Chen, X. Ma, K. He, L. Wang, X. Dai, Z. Fang, X. Xie, X. L. Qi, C. X. Liu, S. C. Zhang, and Q. K. Xue, Phys. Rev. Lett. 105, 076801 (2010).
${ }^{19}$ Y. C. Chang, J. N. Schulman, G. Bastard, Y. Guldner, and M. Voos, Phys. Rev. B 31, 2557 (1985).

${ }^{20}$ O. A. Pankratov, Semicond. Sci. Technol. 5, S204 (1990).

${ }^{21}$ A. Roth, C. Brüne, H. Buhmann, L. W. Molenkamp, J. Maciejko, X.-L. Qi, and S.-C. Zhang, Science 325, 294 (2009).

${ }^{22}$ C. Brüne, C. X. Liu, E. G. Novik, E. M. Hankiewicz, H. Buhmann, Y. L. Chen, X. L. Qi, Z. X. Shen, S. C. Zhang, and L. W. Molenkamp, Phys. Rev. Lett. 106, 126803 (2011).

${ }^{23}$ X. Dai, T. L. Hughes, X.-L. Qi, Z. Fang, and S.-C. Zhang, Phys. Rev. B 77, 125319 (2008).

${ }^{24}$ L. A. Ponomarenko, R. Yang, R. V. Gorbachev, P. Blake, A. S. Mayorov, K. S. Novoselov, M. I. Katsnelson, and A. K. Geim, Phys. Rev. Lett. 105, 136801 (2010).

${ }^{25}$ W. Shan, H. Lu, and S. Shen, New J. Phys. 12, 043048 (2010).

${ }^{26}$ M. Tahir and U. Schwingenschlögl, Appl. Phys. Lett. 101, 013114 (2012). 